\title{
ASYMPTOTIC VALUES OF MEROMORPHIC FUNCTIONS OF SMOOTH GROWTH
}

\author{
by J. M. ANDERSON
}

(Received 2 March, 1978)

1. Introduction. Let $f$ denote a function, meromorphic in $\mathbf{C}$. The question of when a deficient value of $f$, in the sense of Nevanlinna, is an asymptotic value has recently received some attention (see e.g. Hayman [6]). We assume acquaintance with the standard notation of the Nevanlinna theory ([5] Chapter I) which we use without further mention. The following two theorems are known ([1] Theorem 4, and [6] Corollary 2).

THEOREM A. Suppose that $f$ is meromorphic in $\mathbf{C}$ and such that

$$
T(r, f)=O(\log r)^{2} \quad(r \rightarrow \infty) .
$$

If $\delta(a, f)>0$ for some $a \in \mathbf{C}$ (there can be at most one such value a) then, for almost all $\boldsymbol{\theta}$

$$
\liminf _{r \rightarrow \infty} \frac{1}{T(r, f)} \log \left|\frac{1}{f\left(r e^{i \theta}\right)-a}\right| \geqslant \delta(a) .
$$

In particular, since $T(r, f) \rightarrow \infty$ as $r \rightarrow \infty$ we see that $f\left(r e^{i \theta}\right) \rightarrow a$ as $r \rightarrow \infty$, for almost all $\boldsymbol{\theta}$.

THEOREM B. Suppose that $f$ is meromorphic in $\mathbf{C}$ and satisfies

$$
T(2 r, f) \sim T(r, f) \quad(r \rightarrow \infty) .
$$

Then a deficient value of $f$ (there is at most one) is an asymptotic value.

Hayman's results are more far-reaching than is indicated by Theorem B. Functions satisfying the smoothness hypothesis of Theorem B have order zero, but Hayman has a more general form of smoothness which yields results for functions of positive order ([6] Theorem 2). It is easy to see that (1) implies (2).

Theorem $A$ is best possible in two senses. Hayman has shown ([6] Theorem 1) that given any $\phi(r)$ tending to $\infty$ with $r$ there exists an $f$, meromorphic in $\mathbf{C}$, such that $\delta(\infty, f)=1$, while $\infty$ is not an asymptotic value of $f$, and such that

$$
T(r, f)=O\left[\phi(r)(\log r)^{2}\right] \quad(r \rightarrow \infty) .
$$

Also Goldberg and Yeremenko [3] have constructed an entire function $f$ (i.e., with $\delta(\infty, f)=1$ ) satisfying (3) such that if $\Gamma$ is any path extending to infinity on which $f(z)$ tends to $\infty$ with $z$ (there must exist such paths since $f$ is entire) then $\ell(R, \Gamma) \neq O(R)(R \rightarrow \infty)$. Here $\ell(R, \Gamma)$ is the length of the arc $\Gamma \cap\{|z| \leqslant R\}$. Thus the asymptotic path is very far from being a straight line.

Glasgow Math. J. 20 (1979) 155-162. 
Hayman has also shown that, even under the smoothness condition (2) the asymptotic path need not be a straight line ([6] section 4).

2. Results. In this note we shall prove, by a method different from that of Hayman, another theorem for meromorphic functions whose growth exhibits some smoothness.

TheOREM 1. Suppose that $f$ is meromorphic in $\mathbf{C}$, satisfies (2) and $\delta(a, f)>0$ for some a. Then there is a path $\Gamma$ extending to infinity so that

$$
\lim \inf \frac{1}{T(r, f)} \log \left|\frac{1}{f(z)-a}\right| \geqslant \delta(a),
$$

as $z=r e^{i \theta} \rightarrow \infty$ along $\Gamma$, and

$$
\ell(R, \Gamma)=R(1+o(1)) \quad(R \rightarrow \infty) .
$$

In the case when $a=\infty,(4)$ is to be understood as

$$
\lim \inf \frac{1}{T(r, f)} \log |f(z)| \geqslant \delta(\infty) \text {. }
$$

Theorem 1 is clearly an extension of Theorem $\mathbf{B}$, though we emphasise again that Theorem B is a corollary of a more general result of Hayman ([6] Theorem 2). It seems possible that some part of Theorem 1 might be carried over to this more general situation. Theorem 1 also shows that the counter-examples of Hayman, Gol'dberg and Yeremenko cannot satisfy the smoothness condition (2).

It will be clear from our method of proof that radial asymptotic values can sometimes be obtained. We discuss this in $\$ 5$.

3. Proof of Theorem 1. We assume throughout this section that $\delta(\infty)>0$, and shall prove (6). The general result will follow from this by a suitable bilinear transformation. We require the following results for entire and meromorphic functions.

LEMMA 1. If $f$ is an entire function of order zero for which

$$
N(2 r, o, f) \sim N(r, o, f) \quad(r \rightarrow \infty)
$$

then

(a) $n(r, 0)=o(N(r, 0)) \quad(r \rightarrow \infty)$,

(b) $\log M(r, f) \sim N(r, 0) \sim T(r, f) \quad(r \rightarrow \infty)$.

Proof. Part (a) follows immediately from the inequalities

$$
n(r, 0) \log 2 \leqslant \int_{r}^{2 r} \frac{n(t) d t}{t}=N(2 r)-N(r) .
$$


For part (b) we have

$$
\begin{aligned}
N(r, 0) & \leq T(r) \leq \log M(r) \leq r \int_{0}^{\infty} \frac{n(t) d t}{t(t+r)} \\
& \leq \int_{0}^{r} \frac{n(t) d t}{t}+r \int_{r}^{\infty} \frac{n(t) d t}{t^{2}} \\
& =N(r)+o\left(r \int_{r}^{\infty} \frac{N(t) d t}{t^{2}}\right) \quad(r \rightarrow \infty) .
\end{aligned}
$$

Now for any $r_{1}<r_{2}$ and any $\varepsilon>0$ we have (see Hayman [6] Formula 4.5)

$$
N\left(r_{2}, 0\right) \leqslant N\left(r_{1}, 0\right)\left(\frac{r_{2}}{r_{1}}\right) \varepsilon .
$$

It is now easy to see that

$$
r \int_{r}^{\infty} \frac{N(t) d t}{t^{2}}=O(N(r)) \quad(r \rightarrow \infty),
$$

and part (b) follows.

LEMMA 2. If $f$ is meromorphic in $\mathbf{C}$ and satisfies (2) then, for each $a, b \in \mathbf{C} \cup\{\infty\}$,

$$
\max \{N(r, a), N(r, b)\} \sim T(r) \quad(r \rightarrow \infty) .
$$

(For a proof see Valiron [8], Theorème II.).

We also require the following theorem which may be regarded as an analogue of Theorem 1 of [4], but we first introduce some notation.

Definttion. A countable set of circles in the plane is said to form a slim set if the sum of the radii of those circles intersecting the annulus $2^{k} \leqslant|z|<2^{k+1}$ is $o\left(2^{k}\right)$ as $k \rightarrow \infty$.

This definition is to be compared with the definition of an $\mathscr{E}$-set in [4]. A countable set of circles not containing the origin is called an $\mathscr{E}$-set if they subtend angles at the origin whose sum converges. We note that if $\left\{C_{n}\right\}_{n=1}^{\infty}$ is a slim set of circles and if $\delta_{k}$ is the sum of the angles subtended by those $C_{n}$ whose centres lie in the annulus $2^{k} \leqslant|z|<2^{k+1}$, then $\delta_{k} \rightarrow 0$ as $k \rightarrow \infty$. However $\sum \delta_{k}$ may diverge, and it is precisely for this reason that we cannot assert in Theorem 1 that the paths are radial-an assertion which would, in any case, be false ([6] section 4).

THEOREM 2. Let $f$ be an entire function for which

$$
\log M(2 r) \sim \log M(r), \quad(r \rightarrow \infty) .
$$

Then $\log |f(z)| \sim \log M(r)$ as $z=r e^{i \theta} \rightarrow \infty$ outside a slim set.

We also note the following fact about a slim set.

LEMMA 3. If $S$ is a slim set then there is a receding path $\Gamma$ from 0 to $\infty$ lying eventually outside $S$ such that $\ell(R, \Gamma)=R(1+o(1))(R \rightarrow \infty)$. 
Proof. Since $S$ is slim we can, for all sufficiently large $n$, find circles $|z \pm 1|=R_{n}$, neither of which meet $S$, where $2^{2 n} \leqslant R_{n}<2^{2 n+1}$. Also the segment

$$
s_{n}(\theta)=\left\{z: z=R e^{i \theta}, R_{n}-1 \leq R \leq R_{n+1}+1\right\}
$$

can meet $S$ only for a set of $\theta$ whose measure is $o(1)$ as $n \rightarrow \infty$. Thus we can construct a path of the required type as a union of segments $s_{n}\left(\theta_{n}\right)$ where $\theta_{n+1}-\theta_{n}=o(1)$ as $n \rightarrow \infty$, and arcs of $|z \pm 1|=R_{n}$ whose length is $o\left(R_{n}\right)$ as $n \rightarrow \infty$. We choose $|z-1|=R_{n}$ and take $\left|\theta_{n+1}\right|<\left|\theta_{n}\right|$ if $(\pi / 2) \leqslant\left|\theta_{n}\right| \leqslant \pi$ and choose $|z+1|=R_{n}$ and $\left|\theta_{n+1}\right|>\left|\theta_{n}\right|$ if $\left|\theta_{n}\right|<(\pi / 2)$. The path so constructed will have the desired properties.

Thus, if $f$ is an entire function satisfying (7) or (2) then it follows from Theorem 2 that there is a receding path $\Gamma$ such that as $z \rightarrow \infty$ along $\Gamma$

$$
\log |f(z)| \sim \log M(r) \text {. }
$$

This contrasts with a theorem of Essén ([2] Theorem 3) which shows that there is an entire function $f$ satisfying

$$
\log M(r, f)=O(\log r)^{2+\delta}, \quad(r \rightarrow \infty)
$$

where $\delta>0$, and such that for each path $\Gamma$ going out to $\infty$

$$
\lim \inf \left(\frac{\log |f(z)|}{\log M(r)}\right)=0 \text {, }
$$

as $z \rightarrow \infty, z \in \Gamma$.

We assume Theorem 2 and prove Theorem 1 . Since

$$
N(r, \infty, f)<(1-\delta(\infty)+o(1)) T(r, f) \quad\left(r>r_{0}\right)
$$

by assumption, it follows from Lemma 2 that

$$
N(r, 0, f) \sim T(r), \quad(r \rightarrow \infty) .
$$

We write $f(z)=\left(f_{1}(z) / f_{2}(z)\right)$ where $f_{1}$ and $f_{2}$ are entire functions of zero order, having no zeros in common. It now follows from Lemma 1 that

$$
\log M\left(2 r, f_{1}\right) \sim T\left(2 r, f_{1}\right) \sim T\left(r, f_{1}\right) \sim \log M\left(r, f_{1}\right) \quad(r \rightarrow \infty) .
$$

Hence, by Theorem 2 we obtain

$$
\log \left|f_{1}(z)\right| \sim T\left(r, f_{1}\right),
$$

as $z \rightarrow \infty$ outside a slim set. Also, for $|z|=r$ we have

$$
\begin{aligned}
\log \left|f_{2}(z)\right| & \leqslant \int_{0}^{\infty} \log \left(1+\frac{r}{t}\right) d n\left(t, f_{2}\right) \\
& =r \int_{0}^{\infty} \frac{N\left(t, f_{2}\right) d t}{(t+r)^{2}}
\end{aligned}
$$


after two integrations by parts. Hence

$$
\begin{aligned}
\log \left|f_{2}(z)\right| & \leq(1-\delta(\infty)+o(1)) r \int_{0}^{\infty} \frac{T(t, f)}{(t+r)^{2}} d t \\
& =(1-\delta(\infty)+o(1))(1+o(1)) r \int_{0}^{\infty} \frac{N\left(t, f_{1}\right) d t}{(t+r)^{2}} \\
& =(1-\delta(\infty)+o(1)) T(r, f), \quad(r \rightarrow \infty)
\end{aligned}
$$

Thus as $z \rightarrow \infty$ outside a slim set

$$
\begin{aligned}
\log |f(z)| & =\log \left|f_{1}(z)\right|-\log \left|f_{2}(z)\right|>(1+o(1)) T(r)-(1-\delta(\infty)+o(1)) T(r) \\
& =(\delta(\infty)+o(1)) T(r, f),
\end{aligned}
$$

which completes the proof of Theorem 1 .

4. Proof of Theorem 2. Since $f$ satisfies (7) it has order zero and so

$$
\log |f(z)|=p \log |z|+a+\int \log \left|1-\frac{z}{\zeta}\right| d \mu e_{\mathfrak{\zeta}}
$$

where $\mu e_{\zeta}$ is the Riesz mass associated with the zeros of $f$ at points other than the origin. Hence

$$
\log M(r, f) \leq p \log r+a+\int \log \left(1+\frac{|z|}{|\zeta|}\right) d \mu e_{\zeta}
$$

and so

$$
0 \geqslant \log |f(z)|-\log M(r, f) \geqslant \int \log \frac{|\zeta-z|}{|\zeta|+|z|} d \mu e_{\zeta} .
$$

For fixed $z$ let $k$ be such that $2^{k} \leqslant|z|<2^{k+1}$ and write

$$
\int \log \frac{|\zeta-z|}{|\zeta|+|z|} d \mu e_{\zeta}=I_{1}+I_{2}+I_{3}, \quad \text { say, }
$$

where $I_{1}$ is taken over the range $|\zeta|<2^{k-1}, I_{2}$ over the range $2^{k-1} \leqslant|\zeta|<2^{k+2}$ and $I_{3}$ over the range $|\zeta| \geqslant 2^{k+2}$. An estimate entirely analogous to that of $\S 3$ of [4] enables us to conclude that

$$
I_{1}=o(\log M(r, f)), \quad I_{3}=o(\log M(r, f)), \quad(r \rightarrow \infty),
$$

and it remains to deal with the dominant term $I_{2}$. We require the following form of the Boutroux-Cartan Lemma ([4] Lemma 2). 
LEMMA 4. Suppose that $\mu$ is a positive measure in the plane, vanishing outside a compact set, with $\mu(\mathbf{C})=M<\infty$. Then

$$
\int \log |z-\zeta| d \mu e_{\zeta} \geqslant M \log \rho
$$

outside a set of circles, the sum of whose radii is at most $32 \rho$.

We apply this Lemma to $I_{2}$. The total measure in the whole plane is at most $n\left(2^{k+2}\right)$. By Lemma 1 we have

$$
n\left(2^{k+2}\right)=o\left(\log M\left(2^{k}\right)\right) \quad(k \rightarrow \infty) .
$$

More precisely, if we define $\phi(k)^{2}$ by

$$
n\left(2^{k+2}\right)=\phi(k)^{2} \log M\left(2^{k}\right)
$$

then $\phi(k) \rightarrow 0$ as $k \rightarrow \infty$. Thus, by Lemma 4 ,

$$
\begin{aligned}
0 & \geqslant I_{2} \geqslant \int_{0<|\xi|<2^{k+2}} \log |z-\zeta| d \mu e_{\zeta}-\int_{0<|\xi|<2^{k+2}} \log (|z|+|\zeta|) d \mu e_{\zeta} \\
& \geqslant n\left(2^{k+2}\right)\left\{\log \rho-\log \left(2^{k+3}\right)\right\}=n\left(2^{k+2}\right) \log \left(\frac{\rho}{2^{k+3}}\right),
\end{aligned}
$$

outside a set of circles in $2^{k-1} \leqslant|z|<2^{k+2}$, the sum of whose radii is at most $32 \rho$. Choosing $\rho$ so that $\log \left(\rho / 2^{k+3}\right)=(-1 / \phi(k))$ we have

$$
\begin{aligned}
\rho & =2^{k+3} \exp (-1 / \phi(k))=o\left(2^{k}\right) \quad(k \rightarrow \infty), \\
I_{2} & \geqslant \phi(k)^{2} \log M\left(2^{k}\right) \cdot(-1 / \phi(k)) \\
& =o(\log M(r)) \quad(r \rightarrow \infty)
\end{aligned}
$$

Since (9) ensures that the exceptional set of circles forms a slim set, this proves Theorem 2.

5. Radial Asymptotic Values. The principal difference between Theorem 2 and Theorem 1 of [4] lies in the fact that a slim set may extend angles at the origin whose sum may diverge. If this sum happens to converge then the exceptional set is an $\mathscr{E}$-set in the sense of Hayman and we conclude that almost all rays $\arg z=\theta$ will eventually lie outside the set. It is clear that in our situation this will happen if $\phi(k)$, defined by (8), is suitably small.

If $f$ is meromorphic in $\mathbf{C}$ and satisfies (2) and, if, for some $a, \delta(a, f)>0$, then, by Lemma 2

$$
N(r, b) \sim T(r) \quad(r \rightarrow \infty)
$$

and so

for all $b \neq a$.

$$
N(2 r, b) \sim N(r, b) \quad(r \rightarrow \infty)
$$


THEOREM 3. Suppose that $f$ is meromorphic in $\mathbf{C}$ and that $\delta(a, f)>0$ for some $a \in \mathbf{C}$. If

$$
T(2 r, f)-T(r, f)=o\left(T(r, f)(\log \log r)^{-1} \quad(r \rightarrow \infty),\right.
$$

then

$$
\lim \inf \frac{1}{T(r, f)} \log \left|\frac{1}{f(z)-a}\right| \geqslant \delta(a)
$$

as $z \rightarrow \infty$ outside an $\mathscr{E}$-set. In particular $f\left(r e^{i \theta}\right) \rightarrow a$ as $r \rightarrow \infty$ for almost all $\theta$.

Proof. We assume, without loss of generality that $a=\infty$. It follows from (10) that $\delta(b, f)=0$ for $b \neq \infty$. By a theorem of Ahlfors [7, p. 276] we have for all finite $b$, excepting a set of capacity zero,

$$
N(r, b)=T(r, f)+O(T(r, f))^{3 / 4} .
$$

We choose such a number $b$ and write

$$
f(z)-b=\frac{f_{1}(z)}{f_{2}(z)},
$$

where $f_{1}(z)$ and $f_{2}(z)$ are canonical products of genus zero formed with the zeros and poles of $f(z)-b$. We have

$$
\begin{aligned}
n(r, b) & =O\{N(2 r, b)-N(r, b)\}=O\{T(2 r)-T(r)\}+O(T(r))^{3 / 4} \\
& =T(r, f)\left\{\frac{o(1)}{\log \log r}+O(T(r))^{-1 / 4}\right\}=o\left\{\frac{T(r, f)}{\log \log r}\right\} \quad(r \rightarrow \infty) .
\end{aligned}
$$

It follows from (10) that, with the notation of $\$ 4$,

$$
I_{1}=o\left(\log M\left(r, f_{1}\right)\right), \quad I_{3}=o\left(\log M\left(r, f_{1}\right)\right) \quad(r \rightarrow \infty) .
$$

We define $\phi(k)$ by

$$
n\left(2^{k+2}\right)=\frac{\phi(k)^{2}}{\log k} \log M\left(2^{k}, f_{1}\right),
$$

so that $\phi(k) \rightarrow 0$ as $k \rightarrow \infty$, from (10).

Thus $\log \left|f_{1}(z)\right| \sim \log M\left(r, f_{1}\right) \sim T(r, f)$ as $z \rightarrow \infty$ outside a set of circles the sum of whose radii taken over circles lying in $2^{k-1} \leqslant|z|<2^{k+2}$ is at most $O\left(2^{k} \exp (-(\log k) / \phi(k))\right)$ $(k \rightarrow \infty)$. These circles subtend angles at the origin whose sum is

$$
O(\exp (-(\log k) / \phi(k)))=O\left(k^{-2}\right) \quad(k \rightarrow \infty) .
$$

Thus the exceptional set for $f_{1}(z)$ is an $\mathscr{E}$-set. Since, as before,

$$
\log \left|f_{2}(z)\right| \leq(1-\delta(\infty)+o(1)) T(r, f), \quad(r \rightarrow \infty),
$$


we obtain that

$$
\lim \inf \frac{1}{T(r, f)} \log |f(z)| \geqslant \delta(\infty)
$$

as $z \rightarrow \infty$ outside an $\mathscr{E}$-set, and this proves Theorem 3.

I thank Professor W. K. Hayman for his kind help in the preparation of this note.

Note added in proof. Professor A. A. Goldberg of Lvov State University has kindly pointed out to me that Lemma 1 and part of Theroem 2 are contained in the proof of Theorem 3 in the article by A. A. Goldberg and I. V. Ostrovskii entitled "On entire functions and their derivatives of completely regular growth" (in Russian) in the journal "Theory of functions, functional analysis and their applications" Vol. 18 (1973), published by Kharkov State University. I regret that I was unaware of this article while preparing the present paper.

\section{REFERENCES}

1. J. M. Anderson and J. Clunie, Slowly growing meromorphic functions, Comment. Math Helv., 40 (1966), 267-280.

2. M. Essén, Slowly growing subharmonic functions II, to appear.

3. A. A. Goldberg and A. E. Yeremenko, unpublished.

4. W. K. Hayman, Slowly growing integral and subharmonic functions, Comment. Math. Helv. 34 (1960), 75-84.

5. W. K. Hayman, Meromorphic functions (Oxford, 1964).

6. W. K. Hayman, On Iversen's theorem for meromorphic functions with few poles, Acta Math. 141 (1978) 115-145.

7. R. Nevanlinna, Analytic functions (Springer-Verlag, 1970).

8. G. Valiron, Sur les valeurs déficientes des fonctions méromorphes d'ordre nul. C.R. Acad. Sci. Paris 230 (1950), 40-42.

Mathematics Department

UNIVERSTTY COLLEGE

LONDON WC1 